\section{LES PHÉNOMÈNES VOLCANIQUES LEURS CAUSES. - LEURS EFFETS}

(Silite et fin)

\section{\& IV. - Description sommaire de quelques volcans actifs : le Vésuve, le Stromboli et l'Etna.}

D'apıès les détails qui précèdent, il est facile de se rendre comple que, parmi tous les phénomènes que nous présente la nature, il n'en est guère de plus imposant que celui d'un volcan en éruption, si terıibles que soient ses effets. Quoi de plus grandiose, en effet, que celte montagne enflammée qui lance vers le cicl ses nuages ardents, et projette autour d'elle tout ce quelle pent arracher aux profondeurs! Rien au monde ne peut égaler ce spectacle.

Peu de gens, peu de naturalistes même ont eu l'heureuse chance de visiter sans danger des volcans en pleine phase d'aciivité, er d'en ex əminer les divers états. Les rares explorateurs qui se sont hasardés sur leurs pentes ont été bien rarement des á́ologues, et les descriptions uu'ils en ont faites ont été souvent plus fantaisie que réalité. Cependant, des savants éminents, entrautres MM. Fouqué, Lacroix, Vélain, Matteucci, Mercalli, etc., ont pu, dans ces deınières années, suivre pas à pas la marche de plusieurs grandes éruptions, en examiner tous les détails $\epsilon$ t nous retracer leur histoire. La tégion comprise entre Naples et la Sicıle est jusqu'ici celle qui a été le mieux étudíe. car elle permet non seulement d'examiner sans fatigue les mantestations du feu souterrain, mais aussi de recueillir des Éluantillons de laves et de fumerolles, très propres à la détermination de leurs rapports avec les agents internes. Cette ríginn compte actuellement un grand nombre de cratères, et une multitude de petits cônes qui témoignent d'une manière vivante de l'activité du sous-sol. Nous allons donc nous diriger du côlé de Naples et, après avoir fait l'ascension du Vésuve, nous irons, à travers la mer, visiter le Siromboli, puis Volcanellu. En Sicile enin, nous pourrons jouir, en contemplant l'Etna et sa cîme neigeuse, l'un des plus beaux spectacles qu'il soit donné à l'homme d'admirer.

De tous les volcans brûlant à la surface du globe, ancun n'est pl is populaire que le Vésuve. Tour à tour calme et furieux, il est par la nature même de ses éruptions d'une si grande fertilité que les récoltes se succèdent, sur ses pentes, plusieurs fois pendant une même année. La première impression produite sur le voyageur par le Vésuve n'a cependant rien qui inspire la terreur ou provoque l'effroi. Il s'élève solitaire, en effet, au milieu de la plaine qu’il domine, et laisse échapper de son crarère terminal, situé à $\mathbf{~} 200$ mètres environ d'altitude, un mince filet de fumée blanchâtre; il forme un cône à large base, assez régulier, entouré concentriquement, sur les trois quarts de sa surface latérale, par un ancien cratère, la Somma, au milieu duquel il a pris naissance à une époque indéterminée, mais antérieure à notre ère. A deux kilomètres à peine du sommet, à la basc du cône supérieur, se trouve l'observatoire, d'une architecture ausière et solidement établi sur le roc. De la terrasse, on peut contempler le golfe de Naples, le cap Misène, la ville de Pouzzoles, ainsi que les îles de Procida et d'Ischia. Dans les salles de l'observatoire, se trouvent tous les appareils nécessaires au physicien et au météorologiste pour enregistrer les vibrations du sol es prévoir, aulant qu'il est possible, les éruptions. Une très belle collection de laves, représentant toutês les variétés minérales vomies jusqu'ici par la bouche du velcan, est exposée aux yeux des visiteurs et des ascensionnistes. Ajutons à cela qu'un funiculaire, d'une parfaite élégance, mene aujourd'hui presque jusqu'au sommet, et rend ainsil'ascen. sion moins pénible et plus rapide.

- Bien qu'ayant une altitude relativement faible, le Vésuve est. un des volcans les plus puissants que l'on connaisse, et le souvenir de ses grandes crises ne s'effacera jamais de la mémoire des hommes. Tout le pays qui l'entoure est, en effet, bouleversé par les nombreuses éruptions dont il a eu à subir les con- séquences, et par les embrasements souterrains qui ont maintes fois modifié son aspect et sa configuration.

Les Romains savaient dejà̀ que le Vésuve avait été autrefois en activité, mais on habitait cependant sans crainte les villages construits sur ses pentes. «Ces lieux, dit Strabon en parlant de Pompéi et d'Herculanum, sont dominés par le mont Vésuve entouré de riches campagnes, excepté à son sommet, dont la majeure partie offre une surface plane complètement stérile qui a l'aspect d'un monceau de cendres. Au milieu de rochers de couleur sombre qui semblent avoir été consumés par le feu, on aperçoit des couches crevassées. On serait tenté de croire que ces lieux ont brûlé jadis, et qu'jls renferment des cratères où l'incendie s'est éteintfaute d'aliment. » Après un long repos, le Vésuve se réveilla soudainement au mois d'aoùt de l'an 79 , à la suite de tremblements de terre effroyables qui, pendant plusieurs années, ébranlèrent les contrées avoisinantes. C'est dans cette terrible catistrophe que périt Pline l'ancien.

Au cours de cette éruption, Pompér, Herculanum et Stabia furent complètement ensevelies, et un immense champ de laves et de cendres amoncelées recouvrit toute la plaine à l'est du golfe.

Ce n'est que vers le commencement du XVIIIo siècle que l'on a cherché à découvrir les restes te ces villes anéanties. On a pu alors constater la présence de couches épaisses de cendres et de déjections volcaniques formant des assises superposées et, aujourd'hui, rien n'est plus facile au voyageur quue de se promener dans les rues de Pompéi et de pénétrer dans ses palais; il peut de même suivre la grande route garnie de trottoirs qui abrite de nombreux tombeaux, et voir encore la trace antique des chars sur la chaussée pavée de dalles en lave.

Une série de fouilles méthodiquement entreprises ont ainsi permis aux historiens et aux archéologues de retrouver, à travers les couches de cendres en sommeil depuis dix-huit siècles, les plus précieuses révélations pour la science. De ces ruines, on a exhumé les monuments et mille objets d'art et d'industric. Un spectacle des plus saisissants est celui des formes humaines retrouvées pour ainsi dire moulées par la lave; en effet, on comprend facilement que, au cours des éruptions de lave liquide, celle.ci de même que les cendres imbibées d'eau, aient pu envelopper les corps au moment où ils expiraient.

Depuis cette terrible catastrophe, le Vésuve a eu de nnmbreuses et fréquentes éruptions, dont les unes ont causé de grands désastres, et dont les autres soni demeurées relativement calmes. Nous signalerons, tout d'abord, celles des années 204, 5 19, 993, 1036, i 36 . Celle de i 36 fut suriout violente. La plus longue période de repos du volcan fut de 13 I ans, de 1500 a i 1631 , époque à laquelle une grande explosion souterraine causa l'écroulement de la montagne, et produisit une coulée de laves qui alla jusqu'à Portici, en brûlant tout ce qu'elle rencontrait, arbres et maisons, sur son passage. D'autres éruptions eurent lieu également en 1779 et 1861 . Au mois de janvier de 1865 , de véritables décharges électriques se manifesterent au sommet de la montagne, et de nombreux coups de foudre accompagnés de sillons rectilignes ou en zigzags s'élancèrent verticalement du cratère. Comme nous le savons, une éruption assez importante avec émission d'une grande quantité de laves et de bombes eut lieu en 1900 . Enfin, en 1906 , le Vésuve reprit une activité qu'il n'avait jamais eu depuis I'an 79 ; il causa un véritable désordre, non seulement dans la contrée avoisinante, mais même dans des villages assez éloignés de ses pentes. Cette dernière éruption a été étudiée très en détails par M. A. Lacroix.

Depuis cette éruption, le Vésuve est resté relativement calme, bien que de petites coulées se manifestent encore de temps à autre. Par moments, on entend nettement, en s'approchant des bords du cratère, une sorte de bouillonnement continu, et la force d'explosion qui réside à l'jntéricur de sa masse est parfois assez grande pour projeter dans l'atmosphère, jusqu'à une certaine hauteur, de gros blocs de rochers qui, la plupart, retombent bruyamment au milieu de la lave liquide. En maints 
endroits, principalement sur les pentes de la montague, on voit cette lave sourdre à travers les fissures du volcan, et pétiller dans les canaux inclinés; mais, naturellement, la température de cette masse liquide diminue assez rapidement. Si, dans le cratère même elle dépasse souvent I $500^{\circ}$, on peut s'en approcher sans danger lorsqu'elle coule sur les pentes, car elle se meut alors à la façon d'une rivière, et se solidifie en partie à sa surface extérieure et en conservant cependant, grâce à sa très mauvaise conductibilité calorifique, une grande quantité de la chaleur initiale absorbée.

Indépendamment de la lave, le Vésuve lance aussi, hors du cratère, des bombes volcaniques de grandes dimensions et que les Napolitains désignent sous le nom de larmes du Vésuve. On en a vu mesurant plusieurs mètres cubes de volume : on peut dès lors se faire une idée du désastre que peut occasionner, sur une ville, une chute de pareilles masses.

$\mathrm{Si}$, nous éloignant maintenant du Vésuve, nous nous dirigeons plus à l'ouest de Naples, nous pourrons jouir de manifestations naturelles d'un ordre tout différent, et consistant surtout en émanations sulfureuses et en sources chaudes. Une région bien spéciale à ce point de vue, et que les Auciens désignaient sous le noms de Forum de Vulcain ou de Champs Phlégréens (champs brûlants), renferme encore quelques cratères et, à son centre, près de la ville de Pouzzoles, se trouve une solfatare, ou volcan de soufre, dont l'industrie a tiré un grand profit. En maints endroits, le soufre s'y trouve cristallisé en fragments de couleur jaune-citron,"et tout le terrain qui l'environne est composé d'une roche scoriacée et rougeâtre, appelée pouzzolane; cette matière sert aujourd'hui pour l'empierrement des routes et la fabrication des ciments dits volcaniques.

Enfin, à Cumes, non loin du Vésuve, se trouve un sîte plus curieux encore, où les fontaines chaudes exhalent une épaisse fumée. On désigne ces lieux funestes sous le nom d'avernes, parce qu'ils ont été longtemps mortels pour les oiseaux: des gaz délétères ont certainement dû, à une époque plus ou moins rapprochée de nous, se dégager du sol, car, à une faible distance, se trouve la Grotte du Chien dont nous avons précédemment

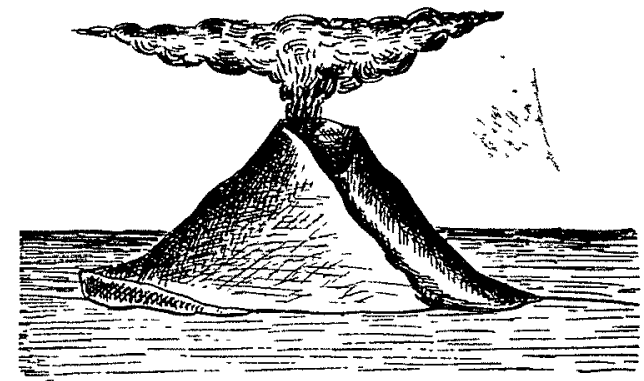

FiG. 7. - Le Stromboli. parlé ; la couche d'acide carbonique y occupe une hauteur de $o^{m} 75$ environ, de sorte qu'un animal de petite taille, un chien par exemple, ne peut y pénétrer sans danger, alors qu'un homme $y$ peut respirer sans malaise. A Royat, dans le Puy-de-Dôme, il existe une grotte du même genre: l'épaisseur de la couche gazeuse s'y modifie constamment, suivant les variations météorologiques, mais d'une façon insensible; elle est généralement voisine de o m. go.

Maintenant que nous connaissons la région vésuvienne, que nous avons visitée et admiré le Vésuve, continuons notre route et dirigeons-nous vers d'autres édifices volcaniques d'une nature différente. En traversant la mer, nous rencontrons sept petites îles, désignées aujourd'hui sous les noms de Lipari ou d'Eoliennes. Placées entre Naples et la Sicile, elles ont joué un grand rôle dans l'histoire des temps mythiques, par les phénomènes météoriques qu'elles ont maintes fois provoqués, et aujourd'hui encore nous les voyons à l'œuvre. Lipari, la plus importante d'entre elles, était déjà célèbre dans l'antiquité par la splendeur de sa capitale et par son grand commerce de soufre. Mais la plus intéressante pour nous est certainement Stromboli, que l'on a souvent regardée comme le phare de la Méditerranée. Cette île compte environ I 500 habitants, groupés presque tous dans la ville d'Inostra; elle est très régulièrement conique (fig. 7), et mesure environ $14 \mathrm{kms}$ de tour; sa hauteur n'est que de $800 \mathrm{~m}$. , mais rien que par sa forme on peut com. prendre combien sa base plonge profondément dans le sol.

Signalé par Homère comme volcan actif, le Stromboli n'a pas cessé de bouillonner jusqu'à nos jours; continuellement il vomit des flammes ardentes et, pendant la nuit, il projette une lumière si vive sur le ciel qu'il paraît en feu. C'est avec un véritable regret, il faut l'avoser, que le géologue quitte ces lieux vivants où l'on contemple la nature à l'œuvre dans toute sa force et dans toute sa splendeur, et où l'on croit momentanément voir revivre ces époques où le globe tout entier, alors qu'il était lui-même un soleil éblouissant, manifestait son incandescence et son énergie d'une façon grandiose. (In ne peut se lasser de contempler ce spectacle vraiment étrange et d'en examiner à chaque instant les phases variées; tout cela excite une curiosité sans cesse croissante qui voudrait pénétrer jusqu'au fond de l'abìme pour y dévoiler le secret de la nature. De nombreux savants ont visité le Stromboli, et tous en ont ressenti les mêmes impressions, Spallanzani, Poulett-Scrope, Charles Sainte-Claire-Deville, Fouqué, Velain, Lacroix, en ont donné des descriptions vraiment dignes d'intérêt.

Le cratère du Stromboli est assez fávorable à l'étude physicochimique des volcans, car on $y$ voit très facilement la lave bouillonner et présenter l'aspect d'une masse luisante, ressen. blant à un bain de métal fondu brillant, même pendant le jout, d'un très vif éclat et s'élevant de quart d'heure en quart d'heure avec un sourd mugissement jusqu'aux bords supérieurs du cratère. De temps en temps, on voit cette lave déborder et se déverser à la manière d'un trop-plein, en s'écoulant jusqu'à la mer par les pentes du volcan. Au milieu des roches scoliacées qui forment ces pentes, on rencontre quelques curiosités naturelles, et principalement des grottes. En particulier, celle qui se trouve sur une petite colline dominant la pointe de Lana est surtout célèbre par ce fait qu'on y peut recueillir en abondance ce joli minéral désigné en minéralogie sous le nom de fer spém culaire, et qui brille comme de l'acier poli.

Mais ce qui a toujours le plus frappé les voyageurs et les géo. logues dans le Stromboli, c'est la continuité de ses éruptions. (*) En effet, tandis qu'on voit des volcans élevés, comme l'Etna, le Cotopaxi, ou l'Hécla, se livrer de temps en temps à des périodes de grande activité en lançant au loin toutes sortes de matériaux, puis redevenir calmes pour un certain temps, au contraire, dans le Stromboli, les forces souterraines sont toujours à l'œuvre, la lave sans cesse bouillante est prête à se déverser au dehors, le cratère toujours est disposé au travail d'éruption; et tout cela sans grand danger, dans la plupart des cas, la lave du Stromboli étant assez fluide pour pouvoir s'écouler sans former bouchon au sommet du cratère, et sans presque jamais provoquer d'ex* plosions dangereuses.

On peut étudier le cratère du Stromboli de deux manières: soit de la mer, à l'aide d'une simple barque, en examinant d'assez près les coulées du volcan, soit sur la montagne ellemême, en s'approchant, autant que cela est possible, du cratère. Il est toujours facile de se rendre compte de la haute tempéra" ture des laves ainsi émises par le volcan, car la plupart des fragments, en arrivant au contact de l'eau, provoquent un bruit aigu semblable à celui d'un fer rouge ou d'un morceau de char bon incandescent que l'on plonge subitement dans l'eau froide,

L'histoire des éruptions du Stromboli se perd dans la nuit des temps, car Strabon, Pline, et les naturalistes anciens, parlent déjà dans leurs écrits de ce volcan qui n'a jamais eu de repos; ils le comparent, au point de vue de son activité, avec ses voisins, et constatent principalement la blancheur de ses feux. La prem mière éruption dont les récits nous ont laissé le souvenir remonte à trois siècles environ avant notre ère. Les historiens du temps d'Auguste et de Tibère nous le dépeignent comme rejetant sans

(*) On a constaté que les principaux tremblements de terre qu'on a observé dans l'Italie méridionale se répartissent à peu près régulièrement suivant un arc de cercle passant par la Sicile et la Calabre, et ayant seth siblement le Stromboli pour Centre. N. D. L. R. 
pitié des matières incandescentes, et une foule de légendes se pittachent à cet abîme dans lequel Grégoire Ier croyait voir le s.jour des damnés.

Mais si le Stromboli jette partout la crainte, il faut reconnaitre qu'il rend des services, car, en plus de sa luminosité constante qui permet aux navires de s'en servir comme d'un pharc, il peut être considéré comme un baromètre d'une grande précision. En effet, il serait soumis, au dire des insulaires, aux variations de l'atmosphère, et les tempêtes, principalement celles causées par les vents du Sud, sont toujours précédées de colonnes de fumée. Les pilotes qui naviguent la nuit se guident ainsi d'après ses flammes, et peuvent juger du temps d'après sa fumée.

Les explosions qui se produisent dans son sein se succèdent parfois avec une si grande rapidité qu'elles ne sont souvent séparées que par des intervalles de quatre à cina minutes seulement. D'après Spallanzani, qui aperçut en partie les bords intérieurs du cratère sans pouvoir y pénétrer, la cavité centrale ne serait pas d'une très grande profundeur. Ln suivant attentivument des yeux les matières lancées à l'extérieur, et en prêtant l'oreille, on constate que les laves, en retombant dans le cratère, produisent un choc qui suit presque immédiatemet leur arrivée dans le gouffre.

Les exhalaisons sulfureuses du Stromboli sont assez nombreuses, car on voit presque constamment une fumée grisàtre acompagnant des projections de toutes sortes, et formant un nuage tantôt vertical tantôt en forme de parasol. Partout où l'on fait un trou dans le roc avec un bâton, il en sort des bouffées de gaz qui ne sont point instantanées et de courte durée, comme on pourrait le croire, mais assez constantes et durables. Sil'on frappe la terre avec le pied, on reconnait une grande résonnance, laquelle n'est ceriainement pras due à la présence de cavités ou de grottes souterraines, mais à la faible densité des laves et à leur structure déchiquetée et caverneusc.

Tout près de cette île se trouve celle de Volcano, possédant une circonférence d'environ i 5 kilomètres et qui s'est accrue par la jonction de l'ile de Volcanello; cette dernière s'éleva tout à coup au sein des eaux, longtemps après que Volcano eût été décrite par Thucydide, historien grec. Elles sont toutes deux très intéressantes à visiter, car la montagne de Volcano se dresse au centre d'une vallée profonde et complètement isolée des plaines environnantes. Son cratère mesure deux kilomètres de circonférence et possède une forme ovale. Elle ressemble extétieurement à un cône droit, et de son sommet on aperçoit parfaitement le fond du cratère ; sur ses pentes, on voit s'échapper, cn de nombreux points, des bouffées de gaz suffocants que l'on ressent même à une assez grande distance.

L'one des curiosités naturelles les plus intéressantes de Volcano est une grotte, située sur la paroi même du cratère, où l'on constate une température élevée, et où l'air est chargé abondam. ment de vapeurs sulfureuses irrespirables. Cette fumée, en se condensant sur les parois intérieures de la grotte, y dépose constamment des sublimations de soufre qui y forment des stalactites en cascade, de toute beauté. Tantôt ce soufre a la couleur de la chair, tantôt il est d'un jaune brillant, à demi translucide sur les parties de faible épaisseur, el d'une grande fraicheur qui indique un grand état de pureté du minéral.

En ${ }_{1727}$, l'ile de Volcano manifesta une tumultueuse colère, car on vit sortir des deux cratères ouverts à son sommet une grande quantité de flammes et de vapeurs accompagnées de bombes volcaniques et de laves embrasées. On entendit à une grande distance un bruit comparable à celui d'une décharge d'artillerie, tandis qu'au fond du gouffre brillait une colline nouvellement formée, couverte de soufre, de pierres rougies par le feu et de lave pâteuse.

Des changements plus oa moins importants se sont successivement opérés dans ce volcan à la suite de grandes éruptions et principalement après celle de 1786 . On entendit, à cette époque, de grands mugissements souterrains accompagnés de formidables tremblements de terre qui firent écho dans toutes les îles Eoliennes; du cratère s'échappa une grande quantité de sable, et l'ouverture qui prit naissance au fond du gouffre changea complètement l'aspect de la montagne et principalement la configuration des pentes du Sud. En r865, Fouqué visita ce cratère, mais, par suite d'une période de calme relatif, il n'untendit point les violentes détonations dont nous venons de parler. Il put cependant constater que dans la chambre intérieure du cratère régnait une tompérature assez élevée; cn plusieurs points de la montagne, là où les fissures laissaient échapper des gaz, on pouvait constater que ces derniers étaient assez chauds pour provoquer la fusion du zinc. Charles Sainte-Claire-Deville avait du reste lui-même remarqué, quelques années auparavant, la présence de flammes bleuàtres dans lintérieur du cratère, capables de fondre le plomb, mais non l'argent.

De même qu'à Stromboli, les matelots de l'île de Lipari consultent Volcann, leur plus proche voisin, avant de se mettre en marche à travers la mer ; ils peuvent prédire à coup sûr, disentils, le beau et le mauvais temps.

Terminons maintenant notre excursion en nous dirigeant vers la Sicile, vers cette île que tant de naturalistes el d'écrivains nous ont dépeinte avec tous ses charmes, et cherchons à contempler son massif dominant, l'Etna. Situé au nord-est de l'ile, cet imposant volcan possède une hauteur de 3.200 mètres environ au-dessus du niveau de la mer, et sa base, nettement limitće par celle-ci et par deux petits cours d'eau, possède une longutur de circonférence de 180 kilomètres; à l'extrémité mérictionale de cette base, se trouve la vieille cité de Catane qui, comme on le sait, a maintes fois été entièrement détruite par le volcan, et a ensuite reparue toujours plus belle et plus sereine $\left(^{\star}\right)$.

L'époque la plus propice aux ascensions de l'Etna est comprise entre le 15 juin et le 15 septembre. De Catane, d'où l'on part généralement pour gravir la montagne, il faut compter environ 36 kilomètres ; on peut aller à dos de mulet jusqu'à la "maison des Anglais", abri assez confortable situé dans une zône absolument déserte, au-dessous du cône terminal.

Pour faire l'ascension de ce dernier, et pour arriver au sommet, il faut ensuive effectuer un trajet des plus pénibles, car les cendres et les scories qui s'éboulent à chaque instant sous les pas des ascensionnistes, les fatiguent énormément et obligent souvent à rebrousser chemin.

Mais lorsqu'on atteint la cime, on se trouve en face d'une réalité si surprenante que l'esprit est sans cesse en proie à un mélange de crainte et d'admiration que rien ne peut égaler. Audessous de soi, on a toutes les îles Eoliennes, Stromboli et Volcano, puis la Sicile elle-même apparaissant dans toute sa splendeur et laissant voir tous ses reliefs et toutes ses vallies, avec ses nombreux cours d'eau dont on peut suivre les mille détours depuis leur source jusqu'à leur embouchure.

Le cône actuel s'élève au-dessus d'une vaste plate forme dont le bord circulaire marque la limite d'un ancien cra'ère, beaucoup plus vaste, mais comblé depuis l'époque de sa formation par les laves et les cendres qui ont jailli de son sein à des époques lointaines. Du reste, on compte aujourd'hui, sur les flancs de l'Ema plus de deux cents couches secondaires qui montrent combien ont été nombreuses, et variées quant à leur résultat, les éruptions qui ont bouleversé cette montagne. Sa cîme fumante est presque toujours couverte de neige, et, parmi les nombreux ravins qui en sillonnent les pentes, on remarque surtout une vallée profonde, désignée sous le nom de Val del Bove, ouverte sur son flanc est, et descendant jusqu'a la mer.

D'après Poulett-Scrope, le Val del Bove proviendrait d'une grande fissure qui aurait été élargie par des torrents impétueux provenant de la fonte subite d'une grande quantité de neige au sommet de la montagne, et due à une éruption instantanée. On raconte, en effet, qu'en 1755 un torrent roula dans cette valléc

“) La catastrophe du décembre 1908 qui a détruit Messine, qui est proche de Catane n'a pas éte accompagnée d'un spasme particuliel de l'Etna. Ce tremblement de terre (ansi que celui tout recent de la Provence) doit se ranger dans la catégorie de ceux qui sont dus à des glissements da couches profondes du sol les unes sur les auties. N. D. L. R. 
avec une vitesse de 2 kilomètres à la minute, entrainant et détruisant tout sur son passage, après avoir donné naissance à de vastes cônes de débris. En 1852 , époque à laquelle se produisit une formidable éruption, un grand nombre d'ouvertures se déclarèrent depuis les régions voisines du sommet jusqu'à la base du précipice qui forme l'entrée du Val. La lave, partant sous forme d'une large nappe à partir du cóne formé près de l'ouverture la plus basse, roula en cascades, et fit entendre dans sa chute un si grand bruit qu'on l'a sozvent comparée à du verre ou à des substances métalliques se brisant avec fracas; l'épaisseur des laves accumulées pendant les neuf mois que dura le cataclysme égala, en certains endroits, cinquante mètres.

Mais, ce qui caractérise surtout l'Etna, c'est la nature et l'aspect de sa lave, ainsi que le mode d'écoulement de celle-ci. En effet, son mouvement de descente peut être assez justement comparé à celui d'une grosse rivière. Lorsque cette masse liquéfiée a coulé pendant un certain temps, elle se fige à sa partie superficielle, et présente alors l'aspect de charbons embrasés s'amoncelant les uns sur les autres. On appelle sciarri les pierres dures et scoriacées qui prennent ainsi naissance pendant le refroidissement de la lave et qui, semblables a ux glaçons qui surnagent sur nos rivières à l'époque des grandes gılées, suivent elles-même un rapide chemin avant de se soliditier en masse.

Pendant l'éruption de 1669 , qui fut célèbre entre toutes et annoncée par des tremblements de terre accompagnés d'éclairs et de coups de tonnerre, la lave accumulée devant le mur de Carane passa pardessus ce dernier et, chose remarquable, ne le renversa point, mais décrivait une courbe à la façon d'une vague sur une digue. Ce phénomène assez original a depuis lors été plusieurs tois constaté,et l'on a trouvé sa raison d'être. En effet, le dégagament subit de gaz au sein de la lave pressée entre le nur et le fleuve liquide possède une force assez expansive pour former une barrière qui permet à la lave de s'elancer veıticalement sans briser les obstacles qu'elle rencontre.

La fertilité des pentes de l'Etna a été connue et appréciée de tout temps, et l'on sait combien la végétation y est florissante. On y distingue trois zónes distinctes : la zône fertile qui donne du blé, des rruits, des herbes aromatiques, de la vigne et de l'huile; la zône boisée où se trouvent d'épaisses forêts; entun la zône aride ou froide qui est d'une triste et constante solitude. Son aspect est noirâtre, ses terres recouvertes de scories et de glaces. A chaque instant, on y voit le feu souterrain et les agents atmosphériques s'unir pour y modifier son aspect.

Dans ses laves, on rencontre un grand nombre de minéraux intéressants et utiles, tels que le cuivre, le mercure, l'alun, le salpètre, l’amiante.

Quelques observateurs ont émis l'hypothèse que l'activité de ce volcan avait été autrefois plus grande que de nos jours, car les auteurs anciens semblent le regarder comme un corps animé d'une extrême vigueur. D'après Pindare qui, le premier, a parlé de ses éruptions, Jupiter aurait enseveli vivants sous la montagne les géants Encelade et Tiphon dont les efforts pour se dégager causeraient les secousses de l'île. Quand " toute la Tricanie tremble et quand le ciel se couvre de fumée brùlante ", ce serait Encelade qui,

" La bouche haletante et le sein enflammé,
"Soulève le fardsau dont 1 l est opprimé

Le 5 mai i go8, l'Etna s'est réveillé partiellement. Des tremblements de terre, dans la région qui l'entoure, se sont fait resscntir avec force, principalement à Milo et à Santa-Severina. Des maisons ont été lézardées, et la panique a été telle que les populations terrorisées quittèrent leurs maisons et allèrent coucher en plein air. Après la formation rapide de trois courants de lave, celle-ci finit par s'arrêter. De la fumée seulement continua à s'échapper des nouvelles bouches, tandis que du fond de la montagne, les grondements disparaissaient lentement.

L'opinion générale qu'ont tous les peuples sur les volcans est due àce fait que les édifices volcaniques sont des appareils d'une très grande puissance comparativement aux moyens que nous possédons pour les combatre. Nous devons, malgré cela, reconnaître que si le volcan cause parfois bien des désastres, et laisse partout une idée de destruction et de ruines, son influence a parfois un retentissement heureux sur la nature du terrain qu'il met en mouvement ; il se charge, en effet, àlui seul, d'apporter à la terre de nouvelles couches fécondantes où brillera ensuite la végétation la plus luxuriante. C'est ce qu'ex. primait Delille avec beaucoup de justesse, quand il écrivait que les volcans,

$$
\begin{aligned}
& \text { "Mais aussi quelquefors hardis fabricateurs, } \\
& \text { "Melent de grands travaux à d'horribles ravages ". }
\end{aligned}
$$

Quoì qu'il en soit, et c'est la dernière conclusion de notre étude, il est certain que nous devons nous incliner à la vue de ces phénomènes. Le monde où nous vivons ne nous est conu que par nos sens; et une si faible partie seulement nous en est accessible que c'est faire preuve d'orgueil, et non de science,que de vouloir chercher à saisir tous les phénomènes qui s'accom. plissent dans l'univers.

Le globe terrestre, soleil éteint, terme partiel d'une évolution cosmique, est comme un immense champ dexpériences où $5 t$ sont accomplies et s'accomplissent encore des actions chimiques aussi variées que nombreuses. Par sa nature même, et par l'harmonie de ses fonctions, il nous permet d'entrevoir quelle a pu être son origine, en rapport avec les causes qui ont aussi donné naissance aux milliers d'astres qui l'environnent. Il nous permet aussi de formuler quelle pourra être sa fin, résulat naturel de tout être qui naît. vit et meuıt. Mais s’il est possible d'arıiver ainsi, par déductions, à cette hypothèse plausible tt évidente, par contre, son origine première restera toujours pour l'homme mystérieuse et cachée.

N'oublions pas que notre intelligence est bornée dès qu'elle cherche à planer au-dessus de l'intini, et que lo probleme des causes premières est de ceux qu'elle est incapable de résoudre.

Jean Escard

\section{ÉTAT ACTUEL de L’ÉLECTROCHIMIE CONSIDÉRATIONS THÉORIQUES}

Communication présentée au Congrès de Marseille, par M. Ootar Donx-Hénautr, protesseur d'Electrochmo à l'Ecole des Mines el a la Faculté Polytechnique du Hanaut, à Mons (Belgique).

Quand on considère la manière d'agir du courant dans les pro cédés électrochimiques, on a coutume de les diviser en procidés électrothermiques et procédés électrochimiques proprement dits.

bans les premer's, le courant ne sert, comme l'on saut quit developper les tempéralures très élevées auxquelles se manifestenl et peuvent se pour'survre les réachons chıniques cherchées ; lo courant n'est qu'une source de chaleur. La thérle de ces opertr trons rentre par conséquent dans les cadres très vastes de la Thermochmie, de la Slatıque el de la Dynamıque chmmiques dont lólectricilé peut ètre exclue. Ce n'est donc pas le lieu d'aborder icı le développement des théortes relatives à ces prósédés.

Dans les seconds, au contraire, le courant électrique est employé à provoquer au sein d'un mılıeu liquide variable des réder trons chimirques correspondant à une transformation spécifique de l'électrıcıté en énergie chimıque.

Il s'agit de l'Llectrolyse et de ses lois : le développement de ces thévies et leur élat actuel rentrent evidomment dans le pro* gramme de ce Congrès et nous les aborderons ici ; mais, tenant compte du but surlout pralıque poursuivı par cette réunion, nous. leur réservorons un examen sommaire afin de pouvolr traiter avec quelque élendue, les procédéls industriels.

ll est utile, pensons-nous, de relier ces problèmes teclmiques à la scıence physıco-chimique en général. Nous négligerons, tout tefois, l'électrolyse des sels fondus dont les applications d'ordre métallurgique n'ont pu elre prises en considóration dans notre travail.

Lorsqu'une substance se dissout dans l'eau en engendrant un systèmc conducteur (électrolyte), une métamorphose s'acconplit dans le système, et cette métamorphose est l'origine de la conduc- 